XIX.

Aus der Kgl. Psychiatr. und Nervenklinik der Universität Königsberg. (Direktor: Geh. Med.-Rat Prof. Dr. Meyer.)

\title{
Die Pathogenese der Psychosen im Lichte der Abderhalden'schen Anschauungen.
}

Von

Dr. Max Kastan,

I. Assistent der Klinjk.

Zwei Faktoren sind es, welche dem Einzelfall ein besonderes Gepräge gegenüber allen anderen mit gleicher Diagnose verzeichneten verleihen: Erstens die Aktionskraft der einwirkenden Schädigung, welche bedingt ist durch deren physikalische und chemische Eigenschaften, zweitens die Reaktionsbreite des Organismus, welche abhängt von seinem: Gesamtzustande in dem Augenblick, in dem die Schädigung ihn trifft. Hat das für die anderen medizinischen Disziplinen nur die Bedeutung: eines intensiven Cnterschiedes - wie wir etwa rom letalen und rom abortiven Typhus sprechen -, so gewinnt dieser Unterschied für die Psychiatrie dadurch an Bedeutung, dass auch die krankhaften Erscheinungen extensiv sehr ron einander abweichen. Alles, was Sache des Temperaments ist, Einfluss des Milieus, Bahnung genannt wird, auch z. B. die Bildungsmöglichkeit individualisiert die Erscheinungen bei jedem einzelnen Kranken ganz erheblich. Aber nicht nur mit Krankheitssymptomen reagiert der Organismus, sondern auch mit Massregeln, sich der Schädigung zu erwehren. Dazu gehört unter anderem die Bildung: von besonderen Abwehrfermenten, deren Nachweis und Auffindung von Abderhalden in ganz neue Bahnen geleitet wurden.

Eine fast verwirrende Fülle von Befunden ist mit Hilfe der Abderhalden'schen Methoden von den Psychiatern erhoben worden, und es erscheint an der Zeit, hier mit sichtender Hand einmal systematisch zu ordnen. Wenn man die Abbauvorgänge in Beziehung setzt zu den psychotischen Erscheinungen, so lassen sich drei wesentlich verschiedene Gruppen erkennen, nämlich erstens Abbauvorgänge, welche ich koordiniert oder pathognostisch nennen möchte, zweitens die super- 
ordinierten oder pathogenen, die die Psychose verursachen, und drittens die subordinierten oder pathogonen Spaltungsprozesse, die durch die Psychose bedingt sind.

$\mathrm{Zu}$ den koordinierten gehört immer der Abbau von Gehirnsubstanz; denn wo Gehirnsubstanz abgebaut wird, hat gleichzeitig eine Schädigung der Hirnfunktion stattgefunden. Nur in dieser Form, nicht etwa umgekehrt, hat der Satz Gültigkeit. Wir kennen auch psychische Krankheiten, bei denen jeder Abbau von Gehirneiweiss fehlt. Das entspricht ganz den anatomischen Befunden Alzheimer's, der deutlich den Abbau der protoplasmatischen von dem der lipoiden Substanz unterscheiden konnte. Was heisst es denn überhaupt, wenn wir sagen Gehirn wird abgebaut? doch nichts mehr und nichts weniger, als dass das Gehirn kein gegenüber dem übrigen Organismus abgeschlossenes Organ mehr bildet; dass seine Eiweissanteile in das Blut übertreten. Der Abbau ron Gehirn wird daher bei einer ganzen Reihe von. Psychosen festgestellt. In erster Linie bei der Paralyse, bei der aúch eine Anzahl anderer Organe die positive Abderhalden'sche Reaktion zeigen. Bei ihr gehören alle Abbauerscheinungen der Gruppe der koordinierten an, denn meiner Meinung nach ist die Paralyse eine ohne spezifische vorausgegangene Infektionen denkbare Allgemeinerkrankung mit exquisiter und dominierender Beteiligung des Gehirns ${ }^{1}$ ). Hervorzuheben ist gerade inbezug auf therapeutische Massnahmen, dass Mayer einen sehr hohen Prozensatz von Leberabbau bei seinen Paralytikern fand. Wir werden nämlich nicht zu einem entscheidenden Urteil darüber kommen können, welches Organ die vermittelnde Rolle spielt zwischen der Lues und der Paralyse, wenn wir nur Patienten untersuchen, bei denen die Paralyse schon ausgesprochen ist. Wir müssen Luetiker vom Augenblick des Eintritts in eine Behandlung an dauernden Kontrolluntersuchungen des Blutes unterziehen und werden dann vielleicht finden, dass eine Anzahl von Fällen, welche später an Paralyse erkranken, vorher Abbauerscheinungen irgend eines Organes aufweist. Vielleicht wird man, wenn man bei noc̀ nicht ausgebrochener Paralyse im Blute von Luetikern Leberabbaufermente nachweist und dann eine besonders energische antiluetische Kur einleitet, die Paralyse bekämpfen können, allerdings halte ich diese Möglichkeit für ziemlich unwahrscheinlich. In der Frage der Entstehung der Paralyse haben ja gerade die beiden eingangs erwähnten Faktoren den Standpunkt für zwei entgegengesetzte Meinungen abgegeben, indem die einen Forscher eine besondere Art des Krankheitserregers, die

1) Anm. b. d. Korrektur: Im Gegensatz hierzu wäre nach Neue die Lues cerebri mit einer Dysfunktion des Gehirns allein verknüpft. 
anderen eine besondere Disposition des Organismus annahmen, während in allerneuester Zeit Forster insofern eine vermittelnde Stellung einnimmt, als er meint, dass der Organismus die Spirochäten umwandelt, bevor die Paralyse zum Ausbruch kommt. Ich glaube, dass man annehmen kann, dass es besondere arsen- und quecksilberfeste Spirochätenstämme gibt, welche gewöhnlich zu den sogenannten refraktären Fällen von Lues führen, bei besonderer Disposition aber, die Metalues verursachen. Ich halte auch das Chronischwerden der Meningitis nicht, wie Nonne, für die conditio sine qua non Paralyse, sondern für den Ausdruck eben jener Disposition, dank welcher der Organismus die akute Meningitis nicht zu überwinden vermag.

Die grösste Anzahl der Autoren beschäftigte sich mit den Abbauverhältnissen bei der Dementia praecox. Hier handelt es sich mit Ausnahme des Abbaues von Gehirn um pathogenen Abbau von Keimdrüsen und in manchen Fällen, die wir wohl hauptsächlich als katatonische anzusprechen haben, von Schilddrüse ${ }^{1}$ ). Es fragt sich nun, ob wir berechtigt sind, eine bestimmte Dysfunktion dieser Drüsen anzunehmen. Bevor wir uns aber in eine Erörterung darüber einlassen, müssen wir uns klar werden über die Funktion der Keimdrüse. Vorausschicken will ich, dass Fauser eine Geschlechtsspezifizität finden konnte ${ }^{2}$ ), einen Vorgang, der wohl seinen Grund in der Körperfremdheit der Zellen gegenüber dem Serum anders geschlechtlicher Personen hat und der wohl gleichzusetzen ist der Unfähigkeit des Körpers, im Gegensatz zu anderen Zuckerarten die allzu fremdartige Raffinose abzubauen.

Die Keimdrüsen haben zweierlei Funktionen. Die innere Sekretion der Sexualhormone wird in der Pubertätszeit unter physiologischen Verhältnissen manifest, weil dann die Epiphyse einen Teil ihrer Funktion aufgibt. Wird die Epiphyse schon vorher durch zystische Gebilde oder durch Geschwülste zersıört, so kommt es schon in der Kindheit zum Auftreten der sekundären Geschlechtscharaktere, Wuchs der Crines, zu Kolostrumabsonderung, zu Mammahypertrophie, zu Erektionen, zu.Priapismus und zu einer frühzeitigen Reife des gesamten Denkens, nie aber etwa zu einer Ejakulation oder Ovulation. Nächst der Epiphyse geht während der Pubertätszeit noch eine andere Drüse, die Thymus, zugrunde, während gerade zu dieser Zeit die Keimdrüsen anfangen

1) Anm. b. d. Korrektur: Auch Nebenniere wird hin und wieder abgebaut. Doch ist auch hier weder Gleichzeitigkeit noch Parallelismus vor allem mit Schilddrüsenabbau durchwegs festzustellen.

2) Anm. b. d. Korrektur: Vielleicht lässt sich die Theorie der Geschlechtsspezifizität auch auf Prostata und Uterus ausdehnen. Das Serum seniler Frauen wenigstens baute nur Uterus, nie Prostata nach meinen Versuchen ab. 
Spermatozoen und Ovula zu sezernieren, d. h. es beginnt die äussere Sekretion der Keimdrüse. Nun ist die Thymus sehr reich an nukleinund phosphorsäurehaltigen Substanzen, die wir in grossen Quantitäten in den Ovula und in den Köpfen der Spermatozoen wiederfinden. Mleiner Ansicht nach besteht hier ein ursächlicher Zusammenhang zwischen dem scheinbaren Untergang der Thymus und dem gleichzeitigen Beginn der äusseren Sekretion der Keimdrüsen, der für die Genese der Dementia praecox von Wichtigkeit zu sein scheint. Ging die bisherige Anschauung über diese Krankheit von der Ueberlegung aus, dass eine grosse Zahl von Fällen im Pubertätsalter erkrankte, so liegt, wie ich glaube, das Entscheidende darin, dass nur ausnahmsweise die Erkrankung vor der Pubertät beginnt. Die von Raecke und Holländer mitgeteilten Fälle beginnen auch in einem Alter, wo die Thymus bereits einer Destruktion anheimfällt. Fälle, die im eigentlichen Kindesalter beginnen, sind nur von Vogt mitgeteilt worden. Man muss sich wohl vorstellen, dass hier schon vorzeitig eine Zerstörung der Thymus sich entwickelt hat ${ }^{1}$ ). Der Organismus bedarf nun dauernd grosser Mengen von Phosphor und Nukleinsäure, und namentlich in der Pubertätszeit. Ist nun die Nachfrage des Organismus grösser als das mit der Nahrung: zur Verfügung gestellte Angebot, so wird er zu der Phosphor- und Nukleinsäure greifen, welche von der Thymus zu den Keimdrüsen transportiert und für die Zwecke der Keimdrüsen, um mit Abderhalden zu sprechen, mundgerecht gemacht worden ist. Diese behalten, obwohl sie biologisch keimdrïsenzelleigen geworden sind, die physiologischen Eigenschaften der Thymus und der Nukleinsäure bei. Vogt and Klose haben an thymektomierten Tieren gezeigt, dass das Hirn schwoll, und dass die motorischen Ganglienzellen eine erhöhte elektrische Erregbarkeit aufwiesen. Der erste Vorgang ist auch bei der Dementia praecox bekannt; Untersuchungen, die augenblicklich von Goldstein in der hiesigen Klinik unternommen werden, haben ebenso ferner die erhöhte Ansprechbarkeit der Dementia praecox-Kranken für den elektrischen Strom fast überall nachweisen können. Es ist selbstverständlich, dass nicht nur die Zellen, welche motorische Leistungen, sondern auch die, welche psychische Leistungen auszuführen haben, krankhaft verändert werden. Führten Vogt und Klose ihren Versuchstieren Kalk zu, so milderten sich die Erscheinungen, um bei Darreichung von Nuklein wieder in erhöhtem Masse aufzutreten. Vogt meint daher, dass die

1) Anm. b. d. Korrektur: Dementia praecox-Krante im Kindesalter sind noch nicht untersucht worden; es wäre interessant zu erfahren, ob ihr Serum auch Keimdrüsen oder etwa Thymus abbaut. Sehr auffällig war bei 2 Dementia. praecox-Seltionen der Befund einer ausgesprochenen Thymuspersistenz. 
Nukleinsäure in der Thymus zu ungiftigen Nukleoproteiden synthetisiert wird und dass bei Wegfall der Thymus eine Ueberschwemmung mit giftiger, weil nicht synthetisch verarbeiteter Nukleinsäure eintritt. Die anatomischen Befunde an den Ganglienzellen, die wir Alzheimer verdanken, zeigen in den ersten Stadien eine lipoide Degeneration: ob diese zustande kommt durch die Umwandlung neutraler Fette, deren Vorhandensein im normalen Gehirn bisher nicht sicher nachgewiesen werden konnte, zu Lipoiden unter N-Aufnahme aus der Phosphor- und Nukleinsänre oder durch Absättigung vorher ungesättigter Phosphatide, vermag ich natürlich nicht $z u$ entscheiden. Später kommt es zu einer Demineralisation (Hamel), zum Mangel an Kalk, der ja nach den erwähnten Versuchen als Gegengift des Nukleins verbraucht werden könnte, und anatomisch zur Fettsackbildung an den Ganglienzellen (Alzheimer, Cotton). Nun haben Aden und Yokohama feststellen kömen, dass Thymus eine hypotonisierende Wirkung ausübt, deren Schädlichkeiten durch vikariierende Adrenalinämie abgewendet werden. Was wir häufig bei der Dementia praecox finden, - ich erinnere nur an Wernicke's akinetische Motilitätspsychose - das lässt auf einen Hypotonus des Vagus schliessen; beschrieben sind Schweissausbruch (Antheaume und Mignot), Pulsbeschleunigung, andererseits aber auch wieder zu anderen Zeiten bei denselben Kranken Pulsverlangsamung und Hauttrockenheit, wie auch schon Trepsat von einem Pseudoödem und Kraepelin von myxödematösen Hautveränderungen der Katatoniker spricht. Es ist nun interessant, dass Wegener, der seine nach der Abderhalden-Methode untersuchten Kranken am besten beschrieben hat, mitteilt, dass gerade die Form, die mir katatonisch za sein scheint, den Abbau von Schilddrüse aufweist. Es muss sich hier um eine dem Myxödem ähnliche Dysfunktion der Schilddrüse handeln. Die Pupillensymptome der Dementia praecox kommen durch die Affinität des Adrenalins, das rikariierend auftritt, zum Sympathikus zustande.

Nun ist es auffällig, dass oft die Dementia praecox erst zum Ausbruch kommt, wenn eine auslösende Ursache vorhanden ist. In der grossen Mehrzahl besteht diese auslösende Ursache in Paerperium und in der Gravidităt. Für diese beiden Fälle könnte ja eine ausreichende Begründung gesucht werden in dem osteomalazischen Stoffwechsel der Graviden und der Puerperalen, den Haberkant annimmt; auch KieweNebenius führt Osteomalazie und Dementia praecox auf dieselbe Ursache zurück. Man muss jedoch die obige Annahme fallen lassen, da die Dementia praecox ausgelöst werden kann auch durch Unfälle, durch Infektionskrankheiten und ähnliches. Vielleicht kann man die Annahme 
gelten Iassen, dass bis dahin die Fermente die Keimdrüsenbestandteile wirksam bekämpften, so dass das Gehirn nicht beschädigt wurde, dass aber der Organismus neue Fermente frei machen musste und dass er in dem Kampfe gegen zwei Fronten schliesslich unterliegt, da inzwischen das Gehirn von nicht abgebauter Drïsensubstanz angegriffen wurde. Die in der Gravidität vorkommenden Fermente gegen Plazenta sind ja grundlegend gewesen für die ganze Abderhalden'sche Methode. Dass im Puerperalserum Fermente gegen Kasein sich finden, gelang mir in allen Fällen nachzuweisen. Es ist anzunehmen, dass bei Traumen grosse Eiweissmassen von verletzten Organen bezw. von den Blutungen aus in den Kreislauf übertreten, dass bei Infektionskrankheiten die Eiweissstoffe der Bakterien, der erkrankten und geschädigten Organe (Exsudate) die Bildung von Abwehrfermenten hervorrufen, abgesehen davon, dass die Krankheitserreger selbst aus ihrem Nährboden geeignetes, plasmafremdes Material für ihre Erhaltung produzieren.

Auf Grund dieser Ueberlegungen wurde bei einigen frischen Fällen eine Therapie angewendet, nach welcher eine erhebliche Besserung; wenn nicht Heilung eingetreten zu sein scheint. Indem ich annahm, dass die Thymussubstanz als Träger der Phosphor- und Nukleinsäure für den Organismus nötig, aus den Keimdrüsen parenteral bezogen aber schädlich wirkte, habe ich Thymustabletten per os gereicht. Bei einer Kranken, die während der Laktation erkrankte, ging ich davon aus, nach einer Substanz zu suchen, welche die Milcheiweissteile am Uebertritt in das BIut bezw. überhaupt ihre Produktion hinderte. Da die Laktation gewöhnlich bei Eintreten der Gravidität unter Entwickelung des Corpus luteum sistiert, so injizierte ich der Patientin Luteoglandol. Ich möchte diese Tatsache nur verzeichnen, ohne zu behaupten, dass Therapie und Erfolg in einem ursächlichen Zusammenhang ständen.

Es hat den Psychiatern immer Schwierigkeiten gemacht, im Anfang: der Erkrankung zu entscheiden; ob bei auslösenden Ursachen sich eine Dementia praecox entwickelt oder ob es sich um eine kurzdauernde symptomatische Psychose handelt. Es war immer auffallend, dass, wie verschieden auch die auslösenden Ursachen an sich waren, die symptomatische Psychose stets das gleiche Gepräge hatte und dass sie meistens den Alkoholpsychosen - Delirium, Korsakow'scher Symptomenkomplex, Halluzinosen (der Wöchnerinnen nach Fürstner z. B.) stark ähnelten. Nun kann man feststellen, dass alle Zustände, welche zu symptomatischen Psychosen führen, oft oder immer mit Lipämie oder mit Hyperglykämie einhergehen. Hyperglykämie wies Benthin für die Eklampsie, Liefmann und Stern für die fieberhaften Krankheiten, Borchard und Bennigson bei Urämie and 
bei durch Oedem komplizierter Nephritis, Milchzucker im Blute von Puerperalen Abderhalden und Fodor, Glykosurie Hödke in $60 \%$. seiner frisch Verletzten nach, Laktosurie der Puerperalen war schon längst bekannt. Bei der Cholämie verlegt von Fürth die Ursache der Delirien in die geschädigte Leber, wobei vielleicht auch der Kohlehydratstoffwechsel gestört ist. Schwere Diabetesfälle gehen oft mit Lipämie einher, abgesehen von ihrem erhöhten Blutzuckergehalt. Dass bei kachektischen und fieberhaften Prozessen infolge der Eiweissschmelzung zur Erhaltung des Organismus grosse Fettmassen in Umlauf gesetzt werden, ist selbstverständlich. Bei der Cholämie kommt es vielleicht, da die Gallenbestandteile überall hindringen und nur vom intermediären Gallenkreislauf ausgeschlossen sind, zu einem Angriff auf das Fett in den übrigen Körperteilen. In der Hauptsache wären die genannten Krankheitsprozesse diejenigen, bei denen wir symptomatische Psychosen anzutreffen pflegen. Nun kann es überall zu einer Abspaltung von Alkohol aus dem im Blute kreisenden Fett oder Zucker kommen. Ich betone; es kann dazu kommen, es wird das nur in wenigen Fällen geschehen, in wenigen Fällen ist aber auch der Erschöpfungszustand von der Psychose begleitet. Mithin muss man wohl drei Etappen annehmen, die teils sämtlich, teils. einzeln zurückgelegt werden, wenn die körperliche Erkrankung zursymptomatischen Psychose führen soll. Der Körper, der Mittel und Wege ergreift, um sich der plasmafremden Bestandteile zu erwehren, vergreift sich in der Wahl der Mittel, er kann z. B. statt einer Diastase ein hefeenzymartiges Ferment frei machen; in diesem $\mathrm{Zu}-$ sammenhange erscheint es mir bemerkenswert, dass Sackai bei allen mit Lipämie einhergehenden Krankheiten einen Mangel des fettspaltenden Fermentes und dass auch Caro bei kachektischen Prozessen eine Reduktion der Lipase nachweisen konnte, sodass der Körper genötigt ist, den Kampl gegen die plasmafremden Substanzen mit anderen Mitteln aufzunehmen. Der gesunde Organismus scheint sich anders. zu verhalten. Bei zwei von sechs puerperalen Seren, bei denen wohl entsprechend dem Milcheiweiss and dem Milchzucker auch Milchfett in das Blut übergetreten sein dürfte, glaube ich, eine Vermehrung der Lipase haben feststellen zu können. Die zweite Etappe wäre die, dass ein sonst regulatorisch wirkendes Organ dysfunktioniert, wiez. B. nach meinen Untersuchungen beim Delirium tremens die Leber, drittens wäre eine besondere Disposition des Gehirns in dem Sinne anzunehmen, dass die Ganglienzellen für noch nicht tief genug abgebaute Stoffebesonders chemorezeptiv wirkten, wie dies übrigens für mechanische Reize nach den Untersuchungen von $J_{a c o b}$ erwartet werden darf. 
Es gelang mir auch einen Anhalt dafür zu gewinnen, wie weit mindestens der Alkohol abgebaut werden muss, um das Delirium tremens hervorzurufen. Ein Patient, dessen Delirium bereits abgelaufen war, bekam wieder die typischen Erscheinungen des Deliriums, nachdem ich ihm zufällig eine Hyoszininjektion gemacht hatte. An zwei weiteren. Fällen, in denen ich diese Injektionen mit Bedacht ausfährte, konnte ich ebenfalls das Delirum wieder auslösen ${ }^{1}$ ). Vergleicht man die chemischen Formen des Alkohols und des Tropins, eines Bestandteils des. Hyoszins, so findet man, dass, wenn ein Atom dem Alkohol genommen wird, beide die Gruppen $\mathrm{CH}_{3} \mathrm{CHOH}$ enthalten. Nun wurde an unserer Klinik von Margulies nachgewiesen, dass Deliranten im Anfang der. Erkrankung lichtstarre mydriatische Pupillen haben, wie wir sie sonst nach Atropingebrauch finden. Das Atropin aber hat die gleiche chemische Konstitution wie das Hyoszin. Während beim Alkohol der chronische Alkoholismus die Ganglienzellen der Hirnrinde und der nervösen Endapparate im Irismuskel so empfindlich macht, dass sie mit dem erwähnten Symptom auf die Gruppe $\mathrm{CH}_{3} \mathrm{CHOH}$ reagieren, ist es bei Atropin und bei dem Hyoszin die Verkettung dieser Gruppe als veresterte aromatische Säure mit hydroxylhaltiger Seitenkette, welche die deliranten Zustände und die Mydriasis herbeizuführen scheint.

Beachtet man das Schicksal des Alkohols im Körper von dieser Seite, so kann man auch zu einer Erklärung kommen für die Tatsache, dass bei Epileptikern der Alkohol besonders schädlich wirkt, indem er Krämpfe, Erregungss- und Dämmerzustände auslöst. Treten nämlich die Gruppen $\mathrm{CH}_{3}$ und $\mathrm{CH}$ mit dem Chlor in Verbindung, mit dem bei Epileptikern der Organismus angereichert ist, so kann es zu Verbindungen des Chlors mit dem Kohlenstoff teils vom Charakter des kampferregenden Chlorkohlenstoffes, teils von der Art der mehr narkotisch wirkenden Substanzen der Chloroformreihe kommen.

Es erhebt sich nun die Frage, auf welche Weise eine Anreicherung: mit Chlor im Organismus des Epileptikers stattfindet. Bei allen Epileptikern, welche der genuinen Epilepsie zuzurechnen sind, fand ich in allen Stadien der Krankheit Abwehrfermente gegen Dünndarm. Die Dysfunktion des Dünndarmes - Dickdarm wurde nie abgebaut ${ }^{2}$ - kann eine doppelsinnige sein. Der Dünndarm ist unfähig, die Nahrungsmittel tief genug abzuspalten. Des weiteren lässt er die Spaltprodukte der Nahrungsmittel nicht durch seine Wandung, sodass sie nicht von dem

1) Anm. b. d. Korrektur: Bei Strychnininjection blieb das Delirium aus.

2) Anm.b.d. Korrektur: Ausnahme hiervon machte ein Exhibitionist, der auch Alkoholmissbrauch getrieben hatte. 
Pfortaderkreislauf aufgenommen werden und so der entgiftenden Leber zugeführt werden, sondern erst vom Dickdarm aus durch die Venae haemorrhoidales direkt in den grossen Kreislauf gebracht werden. Nach epileptischen Anfällen und beim petit mal wurde oft Hirnsubstanz abgebaut. nicht jedoch in der Zeit epileptischer Verstimmung. Ob das Chlor nur aus dem Nahrungsinhalt stammt oder ob es auch auf der vom Magen abgesonderten Salzsäure in den Kreislauf gebracht wird unter Umgehang des Pfortadergebietes, vermag ich nicht zu entscheiden. Man wird bei den Epilepsien verschiedenen Ursprungs suchen müssen, ob und auf welche gleichen körperlichen Vorgänge die gleichen klinischen Symptome zurückgeführt werden können. Alkohol-Epileptiker zeigten weder Abbau von Gehirn noch von Dünndarm Im Anfalle selbst entnommenes Blut reagierte an sich schon so stark positiv (vielleicht infolge der Anhäufung der Aminosäuren), dass die übrigen angestellten Reaktionen nicht verwertbar waren.

Bei Anfällen psychogener Art wie überhaupt bei funktionellen Erkrankungen fanden sich keine Abbauvorgänge bei unserem Material.

Auch chronische Alkoholisten (ohe Komphikation) bauten kein Gehirn ab, einige Lunge.

Nunmehr komme ich zur Besprechung der subordinierten Abbauvorgänge, als welche wir meiner Ansicht nach die Befunde beim manisch-depressiven Irresein ansehen müssen. Bei Melancholie fand Kafka den Abbau von Nebennieren, Binswanger Abbau von Leber, Wegner bei Melancholie Abbau von Keimdrüsen. Das erscheint erst als eine widerspruchsvolle Fülle von Befunden, zumal wenn man liest, dass Wegner beim zirkulären Irresein keinerlei Abbauvorgänge hat beobachten können. Doch lassen sich diese scheinbaren Widersprüche vollständig aufklären, wenn man annimmt, dass Wegner unter Melancholie Fälle mit tiefem traurigen Affekt, unter zirkulärem Irresein solche mit heiterem oder ohne tiefer gehenden Affekt versteht, und wenn man sich klar macht, welche Rolle der Affekt für die körperlichen Vorgänge und deren Ablauf spielt. Nach den Untersuchungen Weber's wissen wir, dass in die Bauchgefässe und in alle von der Bauchaorta abhängigen Organe bei unlustbetouten Affekten eine grosse Blutmasse strömt, bei lustbetonten Affekten jedoch in die Extremitäten. Nun nimmt schon Abderhalden an, dass jedes Organ auf eine bestimmte Blutquantität eingestellt ist. Eine Aenderung der Blutmenge würde also zu einer Schädigang des Organs führen. Ausserdem wissen wir durch andere Untersucher (Werschinin), dass einzelne Serumbestandteile die Fähigkeit zu besitzen seheinen, die Zwischenzellensubstanzen zu lockern and so in innigere Berührung mit dem Zellinhalt und den Zellprodukten 
zu kommen, wodurch diese selbst wieder in das Blut übergeführt werden können. Es ist daher klar, dass wir bei Manischen keinerlei Abbauvorgänge konstatieren können. Dass die Abbauvorgänge bei Melancholischen eine direkte Folge der veränderten Blutverteilung, eine indirekte des Affektes sind, geht wohl am besten daraus hervor, dass es mir gelang, mit dem Serum einer Melancholischen alle von der Bauchaorta aus gespeisten Organe, nämlich Pankreas, Leber, Nebenniere, Milz, Niere, Dünndarm, Dickdarm, nicht aber Gehirn und Schilddrüse abzubauen. Man sieht also, dass diese Abbanvorgänge nichts Charakteristisches für die Erkrankung an sich, sondern nur für das Symptom der Depression haben. In der Tat gelang es mir auch bei den Verstimmungen der Epileptiker in gleicher Weise Spaltungsprozesse von Organen festzustellen, die ebenfalls in das Gebiet der Bauchaorta gehören. Zu erwähnen ist noch, dass auch die von Schultze und Knauer festgestellte Glykosurie bei allen ängstlichen Erregungen einer Erklärung zugänglich wird. Kann doch sowohl Adrenalin von der Nebenniere und Glykogen von der Leber aus in das Blut verschleppt werden, während zugleich das Pankreas unter den veränderten Zirkulationsveränderungen leidet.

Für die Differentialdiagnose der Psychosen kommt es demnach darauf an, geeignete Kombinationen auszuwählen, so dass Organe zur Reaktion benutzt werden, deren Gesamtheit bei der einen Krankheitsform die positive Reaktion geben, während sie bei anderen Krankheitsformen entweder negativ reagieren oder positiv nur mit anderen für die betreffende Krankheit nicht in Betracht kommenden Organen zusammen.

Für die Erkenntnis der Pathogenese der Psychosen ist uns durch die Abderhalden'sche Methode und durch die Anffassungen, auf der diese fussen, manche wichtige Handhabe gegeben und viele neue Fragestellungen aufgerollt wurden, wenn auch noch vieles ungeklärt bleibt, z. B. ob es sich um Verschiedenheiten der Lokalisation oder der Disposition handelt, wenn infolge des chronischen Alkoholismus oder der senilen Veränderungen bald diese, bald jene Krankheitsfosm auftritt. Denn es ist klar, dass hierbei grosse Vorsicht bei der Bewertung bei Befunden obwalten muss und dass der von Neue nachgewiesene Abbau von Prostata, Uterus und anderen Organen wohl nicht nur bei senil Dementen, sondern auch bei geistesgesunden Greisen sich wird finden können. 